\title{
Vector Boson Scattering prospects for High-Luminosity LHC at CMS in the WZ final state
}

\author{
Deniz POYRAZ*广 \\ Department of Physics and Astronomy, University of Ghent, Proeftuinstraat 86, B-9000 Ghent, \\ Belgium \\ E-mail: deniz.poyraz@eern.ch
}

Studies of the $p p \rightarrow \mathrm{W}^{ \pm} \mathrm{Zj} j$ vector boson scattering processes in $14 \mathrm{TeV}$ pp collisions using the upgraded CMS detector are presented. These studies include assessments of the expected precision of measuring the electroweak cross sections and the discovery potential for observing longitudinal vector boson scattering. All results are presented with a luminosity of $3 \mathrm{ab}^{-1}$ and comparisons with the non upgraded CMS detector including its ageing due to radiation are performed.

XXVII International Symposium on Lepton Photon Interactions at High Energies 17-22 August 2015

Ljubljana, Slovenia

* Speaker.

${ }^{\dagger}$ On behalf of the CMS Collaboration 


\section{Introduction}

The vector boson scattering (VBS) is a key process to study the electroweak sector of the Standard Model (SM). The vector bosons acquire their mass and longitudinal polarization states through the spontaneous electroweak symmetry breaking. In the absence of the SM Higgs boson, the amplitudes of VBS would increase with energy and ultimately violate unitarity. Therefore VBS cross section measurement is a confirmation of the SM Higgs mechanism and any deviations from the expected values are indications of new mechanisms beyond the SM. This process, currently unobserved, is studied in view of the LHC high luminosity upgrade (HL-LHC). The upgraded detector will run at the centre-of-mass energy of $14 \mathrm{TeV}$ and will deliver $3 \mathrm{ab}^{-1}$ integrated luminosity. Under these conditions the detector will have to stand a significant dose of radiation and will suffer from high pile-up events due to the high luminosity. The basic goal of the upgrade is to maintain the excellent performance of the CMS[1] detector and in some cases, improve the detector performance. Prior to the HL-LHC, several smaller upgrades to the CMS detector will also happen, in the so-called Phase-I upgrade, without modifying much its basic configuration [2]. This Phase-I detector should allow to collect up to $300 \mathrm{fb}^{-1}$ of data. To reach $3 \mathrm{ab}^{-1}$ of collected luminosity, a second upgrade, the Phase-II one, is necessary. In order to assess the sensitivity of the upgraded CMS experiment to the VBS, the precision on the cross section and the expected discovery significance for the longitudinal part of the EWK component cross-section are studied in detail for the different upgrade scenarios explained below.

Phase I: the CMS detector after the Phase-I upgrade assuming no degradations due to radiation and 50 pile-up events per collision on average.

Phase I aged: the CMS detector after the Phase-I upgrade including degradations of performances due to the expected accumulated radiation corresponding to $1 \mathrm{ab}^{-1}$ of data taking and 140 pile-up events per collision on average.

Phase II: the fully upgraded Phase-II CMS detector[3] and 140 pile-up events per collision on average.

The detector response has been simulated parametrically with DELPHES[4] package.

\section{WZ Scattering}

The scattering of vector bosons happens at the LHC when each of the incoming protons of a collision radiate a vector boson. These two then interact with each other, and their decay products and the two quarks deflected from the beam direction called tag jets are detected. For this study, only fully leptonic decays of the vector bosons in WZ final state are selected, each vector boson decaying into electrons or muons, in order to avoid the ambiguities due to the separation of the tag jets from the vector boson decay products. The WZ scattering is identified by two forward highly energetic tag jets that have typical VBS topology with high $m_{j j}$ (greater then $600 \mathrm{GeV}$ ) and have large pseudorapidity difference ( $\Delta \eta_{j j}$ larger than 4), three charged leptons with $p_{T}>20$ $\mathrm{GeV}$ and $\eta<2.4$ where two of them have same flavour, opposite charge with an invariant mass within $\pm 10 \mathrm{GeV}$ around the $\mathrm{Z}$ boson mass, missing transverse energy greater than $30 \mathrm{GeV}$ from the 
undetected neutrino that comes from the $\mathrm{W}$ decay. The background contributions are production of WZ pair via strong interactions, the main background due to high cross section, and ZZ production. The instrumental backgrounds from wrong identification of jets as leptons have been evaluated and found to be negligible in this analysis. The signal and irreducible backgrounds have been generated at leading order with the PHANTOM [5] and MADGRAPH [6] generators. The sensitivity of the measurements are studied in four channels: eеe, $е e \mu, \mu \mu \mu, \mu \mu e$ and the optimisation is done with $1 \mathrm{D}$ and 2D template fit over different kinematic variables. The systematic uncertainties affecting the WZ scattering analysis are listed in Table 1 together with their magnitudes, for each of the scenarios considered.

\begin{tabular}{|l|c|c|c|}
\hline & Phase I & Phase I aged & Phase II \\
\hline jet energy scale & $1-3 \%$ & $1.5-4 \%$ & $1-3 \%$ \\
jet energy resol. & $5 \%$ & $6.5 \%$ & $5 \%$ \\
muon energy scale & $1 \%$ & $2 \%$ & $1 \%$ \\
muon energy resol. & $1 \%$ & $2 \%$ & $1 \%$ \\
electron energy scale & $2 \%$ & $4 \%$ & $2 \%$ \\
electron energy resol. & $2 \%$ & $4 \%$ & $2 \%$ \\
lepton efficiency & $2 \%$ & $2 \%$ & $2 \%$ \\
signal acceptance & $2 \%$ & $2 \%$ & $2 \%$ \\
QCD scale choice & $3 \%$ & $3 \%$ & $3 \%$ \\
parton densities & $7 \%$ & $7 \%$ & $7 \%$ \\
LHC luminosity & $2.6 \%$ & $2.6 \%$ & $2.6 \%$ \\
\hline
\end{tabular}

Table 1: The systematics used for the WZ scattering analyses for each of the detector scenarios considered.

The inclusive EWK cross-section for WZ boson production with two jets is determined by fitting the $2 \mathrm{D}$ distribution of $\left(p t_{j j}, \Delta \eta_{l l}^{S S}\right)$, where $p t_{j j}$ is the transverse momentum of the tag jets pair and $\Delta \eta_{l l}^{S C}$ is pseudorapidity separation between the two leptons of same sign (SS). The expected total uncertainty on the measurement of the EWK WZ scattering cross section combining all trilepton categories is shown in Fig. 1a as a function of the collected luminosity for the three detector configurations. For $3 \mathrm{ab}^{-1}$ of data, uncertainties of $\sim 10 \%$ can be achieved with the Phase I and Phase II detector scenarios, while the Phase I aged detector would provide uncertainties of the order of $12 \%$.

The best expected sensitivity of the longitudinal component of the EWK WZ scattering is achieved with $2 \mathrm{D}$ fit of $\left(\Delta \varphi_{j j}, p_{T}^{\ell_{1}}\right)$ distributions. In this specific case, the event selection is tightened by requiring that the lepton pair associated to the $\mathrm{Z}$ boson has an invariant mass within $5 \mathrm{GeV}$ of the $\mathrm{Z}$ mass. The performances obtained in this configuration, combining all tri-lepton categories is shown in Fig. 1b as a function of the collected luminosity for the three detector scenarios. For the Phase II detector with $3 \mathrm{ab}^{-1}$ of data, an expected significance for the LL component of 1.4 sigmas can be reached while values lower by $\sim 25 \%$ for the Phase I aged detector are expected. 


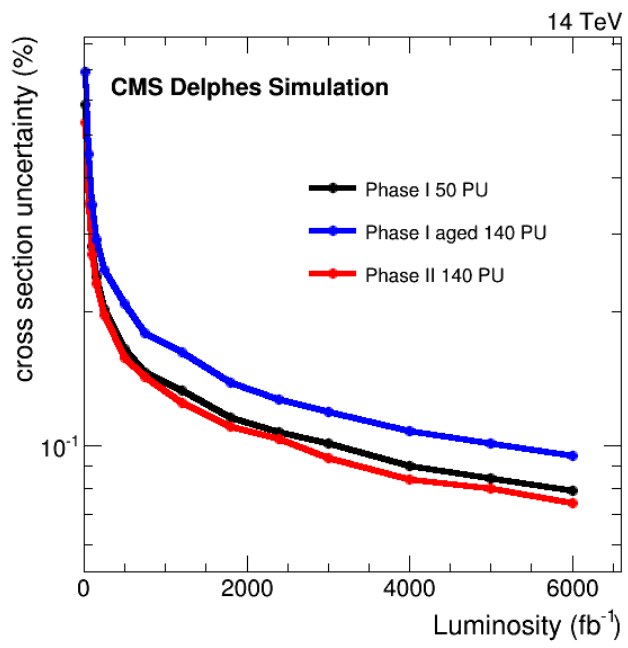

(a) The expected total uncertainty for the various detector scenarios for the EWK WZ cross section measurement as a function of the collected luminosity.

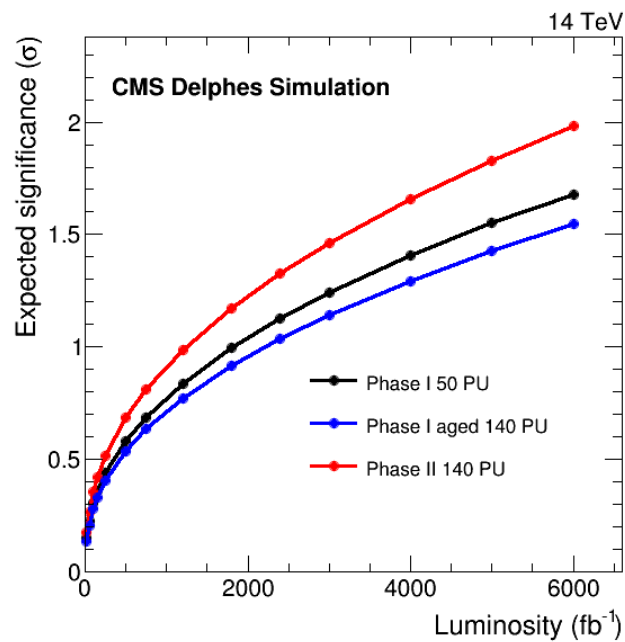

(b) The expected discovery significance for the longitudinal vector bosons scattering for the various detector scenarios as a function of the collected luminosity for the WZ analysis.

Figure 1: Detector performances for the WZ EWK and longitudinal vector boson scattering

\section{Conclusions}

In this study the comparison of the different CMS upgrade scenarios for the HL-LHC are performed. WZ scattering cross section shows that upgraded CMS detector will recover the performances of the current detector due to ageing, and sensitivity to the scattering of longitudinally polarized vector bosons shows upgraded detector over-preforms the Phase I detector.

\section{References}

[1] S. Chatrchyan et al. [CMS Collaboration], JINST 3, S08004 (2008).

[2] Technical proposal for the upgrade of the CMS detector through 2020, CERN-LHCC-2011-006 ; CMS-UG-TP-1 ; LHCC-P-004

[3] Technical Proposal for the Phase-II Upgrade of the CMS Detector, CERN-LHCC-2015-010 ; LHCC-P-008

[4] J. de Favereau et al. [DELPHES 3 Collaboration], JHEP 1402 (2014) 057 [arXiv:1307.6346 [hep-ex]].

[5] A. Ballestrero, A. Belhouari, G. Bevilacqua, V. Kashkan and E. Maina, Comput. Phys. Commun. 180 (2009) 401 [arXiv:0801.3359 [hep-ph]].

[6] J. Alwall et al., JHEP 1407 (2014) 079 [arXiv:1405.0301 [hep-ph]]. 